\title{
Current harmonization activities related to risk/safety assessment by the OECD Working Group on Harmonisation of Regulatory Oversight in Biotechnology
}

\author{
Philip MACDONALD* and Stephen YARROW \\ Canadian Food Inspection Agency, 59 Camelot Drive, Ottawa, Ontario K1A 0Y9, Canada
}

\section{INTRODUCTION}

Current activities at the OECD concerning risk/safety assessments for the environmental release of products of biotechnology are conducted within the Working Group on Harmonisation of Regulatory Oversight in Biotechnology (Working Group). The Working Group consists of individuals from government ministries or agencies that are responsible for the environmental risk/safety assessment of products of modern biotechnology. The work of the Working Group has evolved from considerations of the environmental safety of field trials of GM crops to the consideration of the environmental safety implications arising from the commercialization of GM crop plants. The Working Group concentrates on environmental health and safety and thus complements the work on food and livestock feed safety conducted by the sister group, the Task Force for the Safety of Novel Foods and Feeds.

\section{REGULATORY HARMONIZATION}

A key activity of the Working Group is working towards regulatory harmonization to ensure that the information used in risk/safety assessments, as well as the methods used to collect such information, are as similar as possible. These types of activities are undertaken in order to:

- increasing mutual understanding of risk/safety assessment approaches among member countries;

- share knowledge;

- use resources effectively;

- increase the efficiency of the risk/safety assessment process.

* Corresponding author: pmacdonald@inspection.gc.ca
The desired outcome of successful cooperation towards achieving regulatory harmonization is to improve the ability of member countries to make well developed biosafety decisions. The regulatory harmonization activities that have taken place in the working group have also served to provide a useful example of sound sciencebased regulation of biotechnology in other international discussions.

\section{ACTIVITIES OF THE WORKING GROUP}

The Working Group has focused primarily on the regulatory oversight and risk assessment of products of transgenic crop plants, although important work has also been undertaken on genetically engineered microorganisms, led chiefly by the Sub-Working Group on Micro-organisms. The work to date has focused on three general project areas:

- consensus documents;

- information dissemination;

- harmonizing risk/safety assessment considerations and regulatory oversight.

\section{FACILITATING HARMONIZATION WITH CONSENSUS DOCUMENTS}

A significant output of the Working Group has been the production of consensus documents on the biology of a variety of crop plants and some micro-organisms. Consensus documents include a description of the current uses of the plants or micro-organisms along with a description of the basic biology and taxonomy of the 
species, key physiological characteristics and potential interactions with other species. Those consensus documents concerning crop plants will also indicate areas where the crop is cultivated and the usual associated agronomic practices. These documents are intended to serve as an important base-line for a risk/safety assessment with which to compare the behavior of transgenic forms of the organisms to un-modified forms, and have proven highly useful to risk/safety assessors in both OECD and non-OECD countries.

There Working Group has published 23 consensus documents and there are 15 more in preparation. The documents cover a wide range of crop species and some traits associated with transgenic crop plants. There are also consensus documents on selected micro-organisms and some tree species.

The development of the Points to Consider Document by the Working Group has provided a template that ensures consistent formatting and content of a consensus document. In this manner, the documents have maximum utility. The Points to Consider Document also provides a mechanism to decide on updates of existing documents and to propose consensus documents on species that address the needs of non-member countries.

The Working Group has also developed guidance documents, including: Guidance Document on Methods for Detection of Micro-organisms Introduced into the Environment and the OECD Guidance for the Designation of a Unique Identifier for Transgenic Plants. The unique identifier, a nine-digit alphanumeric code that is given to each transgenic (or genetically engineered) plant that is approved for commercial use, including planting and food/feed use, has been adopted as a mechanism for unique identification by the Cartagena Protocol on Biosafety for use with the Biosafety Clearing House database of the Protocol.

\section{FACILITATING HARMONIZATION BY INFORMATION DISSEMINATION}

Communication between OECD countries and to nonmember countries is a critical component in facilitating harmonization. In keeping with this goal, the Working Group has developed and continues to maintain a database, Biotrack, which includes:

- information on products that have been approved for commercial use, including unique identifiers;

- information related to regulatory developments in OECD member countries;

- information on field trials of transgenic organisms;

- documents produced by the Working Group and the Task Force, such as the consensus documents.
Elements of the Biotrack database interoperate with the Biosafety Clearing House database. The Biosafety Clearing House is a key component in the development and implementation of the Cartagena Protocol on Biosafety.

\section{NEW INITIATIVES TO FACILITATE HARMONIZATION}

Environmental Considerations in a Risk/Safety Assessment of a Transgenic Plant

The two OECD sponsored conferences on LMOs and the Environment (see conference proceedings for the most recent meeting at: http://www.oecd.org/document/ 18/0,2340,en_2649_34387_2509330_1_1_1_1,00.html) have provided a significant contribution to understanding the current state of science on the environmental interactions of transgenic plants and the assessment of their risks. The Working Group sought an avenue to capture the outcomes of this work, and this has lead to a proposal to develop a discussion document on the core considerations for an environmental assessment of a transgenic plant. It was felt that a document of this type could capture the underlying considerations of the environmental risk/safety assessment in order to serve as guidance to regulatory officials on the type of information that can be used to evaluate the differences between the way transgenic plants and their non-transgenic counterpart interact with the environment in managed and unmanaged ecosystems. This evaluation can then be used in assessing the likelihood that the plant will be harmful to the environment, either directly or indirectly. Outlining data needs and acceptable analytical approaches for environmental risk/safety evaluations should also assist in the preparation of data packages by developers of transgenic plants seeking regulatory approval to introduce these plants into agricultural production or commerce.

The development of the document began with the creation of a discussion document for the Working Group that reviewed the criteria employed by different regulatory authorities for the environmental assessment of a transgenic plant. The review highlighted similarities and differences in the assessment criteria in order to provide a basis for the discussion document and to inform the procedure to develop the program of work. A component of the review was a consideration of how different regulatory authorities incorporated deficiencies in baseline data and areas of scientific uncertainty into the consideration of environmental data. After some extensive discussion including a face to face meeting of the countries directly involved in the drafting of the document, a program of work has been developed and an initial draft of the document is underway. 
The proposed document on the Environmental Considerations in a Risk/Safety Assessment of a Transgenic Plant will highlight the importance of the consensus documents in providing important baseline information on the biology and interactions of an unmodified plant in the environment, and these documents can be seen as companion to each other. The Environmental Considerations in a Risk/Safety Assessment of a Transgenic Plant document will capture the general principles developed by the OECD and articulated at the OECD-sponsored meetings on LMOs and the Environment for the environmental assessment of a transgenic plant. It will highlight that specific information may vary with plant species, the specific types of modifications, and end use. This document will concentrate exclusively on the requirements for the environmental assessment of crop plants, and not include information criteria for trees and aquatic plants. Although the intent is to describe fundamental criteria, the document will reaffirm the concept that the risk/safety assessment is conducted on a case-by-case basis.

Molecular Characterization for Transgenic Plants

Similar to the work on the Environmental Considerations in a Risk/Safety Assessment of a Transgenic Plant, the Working Group has embarked on a joint project in collaboration with the Task Force for the Safety of Novel Foods and Feeds to develop a document that describes the elements necessary for the molecular characterization of a transgenic plant. The document is meant to serve as guidance to a risk assessor on how to use submitted molecular characterization data in a risk assessment, and as a tool to identify areas where the risk assessor may require additional data. The document will not contain discussion on which information elements are or are not important to the risk assessment, as this type of consideration will be determined by the competent authority conducting the risk assessment. The document on the Molecular Characterization for Transgenic Plants will include discussion of:

- transformation methods;

- inserted DNA, expressed RNA, expressed protein, post-translational modification;

- inheritance and genetic stability.

This molecular characterization document will complement existing documents, such as the consensus documents, and documents under development, by the Working Group and Task Force and can be applied to a food feed or environmental assessment.

\section{OTHER NEW INITIATIVES}

Consensus document on Atlantic salmon

The Working Group has recently begun work to address environmental risk/safety issues associated with the Atlantic salmon. This is a departure from the traditional concentration of the Working Group on plants and micro-organisms, and recognizes the emerging challenges posed by the possible commercialization of a transgenic fish. The work began with an expert workshop that was held in Moscow in late 2004 on the biology of Atlantic salmon that identified and reviewed the type and availability of baseline information that may be required for a risk/safety assessment of a transgenic fish species. This informed the discussion on the information requirements for the development of an Atlantic salmon consensus document. The abstracts from this meeting are available at: http://www.oecd.org/LongAbstract/0,2546, en_2649_37437_37369197_119829_1_1_37437,00.html

This work continues, and the drafting of a consensus document on Atlantic salmon is proposed as part of the continuing work of the Working Group. In the future, additional projects related to transgenic animals are likely.

\section{CONCLUSION}

The documents developed by the Working Group represent the best efforts of experts from the member countries and provide a useful source of guidance to:

- risk assessor in competent authorities;

- developers of products of biotechnology, as a source of information for applications to regulators;

- regulatory agencies and regulatory officials;

- regulatory policy makers;

- non-member countries, for consideration towards enhancing or establishing regulatory regimes.

The Working Group is geared to towards harmonizing regulatory and risk assessment approaches for products of modern biotechnology. Over its history, the Working Group has produced useful scientific documents that have been recognized for their scientific soundness and applicability. The Working Group is building on its past successes by continuing to produce relevant documents that provide guidance on the risk assessment of products of modern biotechnology. 